\title{
Mesohepatectomy for the treatment of patients with centrally located hepatocellular carcinoma
}

\author{
CHAO-HUI ZUO ${ }^{1,2}$, XIAO-XIN QIU ${ }^{1,2}$, YONG-ZHONG OUYANG ${ }^{1,2}$, DI ZHANG ${ }^{1,2}$, HUA XIAO ${ }^{1,2}$, \\ SHENG-CHUAN MO ${ }^{1,2}$, CHUN-QI TAN ${ }^{1,2}$, MING TANG $^{1,2}$ and HAI-ZHEN ZHU ${ }^{2}$ \\ ${ }^{1}$ Department of Gastroduodenal and Pancreatic Surgery, Hunan Provincial Tumor Hospital \\ and Affiliated Tumor Hospital of Xiangya School of Medicine, Central South University, Changsha, \\ Hunan 410013; ${ }^{2}$ Liver Cancer Translational Medicine Center of Hunan University and \\ Hunan Provincial Tumor Hospital, Changsha, Hunan 410082, P.R. China
}

Received January 4, 2014; Accepted May 27, 2014

DOI: $10.3892 / \mathrm{mco} .2014 .309$

\begin{abstract}
Mesohepatectomy is considered a feasible option for patients with centrally located hepatocellular carcinoma (HCC). However, mesohepatectomy is a technically demanding and less frequently used procedure. In this study, we summarized the surgical experience and evaluated the clinical outcomes of mesohepatectomy in 24 patients with centrally located HCC. Of these patients, 9 were treated with hepatectomy of Couinaud's segments IV, V and VIII with concurrent cholecystectomy; 8 underwent resection of segments IVb, V and VIII, including 7 patients who also received a cholecystectomy; 4 underwent hepatectomy of segments IVa, V and VIII; and 3 patients were treated with hepatectomy of segments I, IV, V and VIII, with concurrent cholecystectomy. The Pringle maneuver was used on 17 patients during hepatectomy. Total hepatic vascular exclusion (HVE) was performed on 3 patients and HVE was not used on 4 patients. The average mesohepatectomy operative time was $238 \mathrm{~min}$ and the average intraoperative blood loss was $480 \mathrm{ml}(200-2,200 \mathrm{ml})$. There was no intraoperative mortality and the postoperative morbidity rate was $25 \%(6 / 24)$. The 1- and 3-year overall survival rates were 76 and $46 \%$, respectively. Therefore, mesohepatectomy is a safe and effective surgical procedure for the treatment of centrally located HCC and HVE during mesohepatectomy for centrally located HCC is crucial to the success of the operation and postoperative patient recovery.
\end{abstract}

Correspondence to: Professor Chao-Hui Zuo, Department of Gastroduodenal and Pancreatic Surgery, Hunan Provincial Tumor Hospital and Affiliated Tumor Hospital of Xiangya School of Medicine, Central South University, 283 Tongzhipo Road, Changsha, Hunan 410013, P.R. China

E-mail: zuochaohui@vip.sina.com

Key words: mesohepatectomy, centrally located, hepatocellular carcinoma

\section{Introduction}

Centrally located hepatocellular carcinoma (HCC) is defined as HCC closely adjacent to the central hepatic segments, including Couinaud's segments IVa, IVb, V and VIII, without usually including malignancies within the caudate lobe (1). Centrally located HCC is adjacent to the portal vein, bile duct, hepatic vein and inferior vena cava. Due to the close proximity to these major vessels, the treatment of such cancers remains challenging. Radiofrequency ablation (RFA) and percutaneous ethanol injection are less effective for larger $(>5 \mathrm{~cm})$ compared to smaller tumors (2-4). Centrally located HCC had long been considered unsuitable for surgical resection and the traditional treatment for such cancers included an extended right or left hepatectomy (5-7). However, extended hepatectomy is associated with higher morbidity and mortality, mainly due to the increased risk of postoperative liver failure $(8,9)$.

Over the last few years, with the advancement in the surgical techniques for liver cancer, mesohepatectomy has become a feasible option for patients with centrally located HCC, particularly for those with post-hepatitis cirrhosis. Previous clinical studies demonstrated that mesohepatectomy may be superior to extended hepatectomy, as it reduces the volume of the resected liver $(10,11)$. However, mesohepatectomy is a technically demanding procedure and may cause injuries to blood vessels and bile ducts, resulting in increased blood loss; therefore, it is less frequently used $(11,12)$ and the application of mesohepatectomy in the treatment of centrally located tumors has not been adequately assessed.

The aim of this study was to review the surgical techniques, clinicopathological characteristics and outcomes of 24 patients who underwent mesohepatectomy, in order to determine whether this treatment is safe and effective for patients with centrally located HCC. We also summarized the surgical procedures and our experience with effective mesohepatectomy for the treatment of centrally located HCC.

\section{Patients and methods}

Patient information. A total of 24 patients, including 19 men and 5 women (mean age, 53 years; range, 27-74 years), with 
A

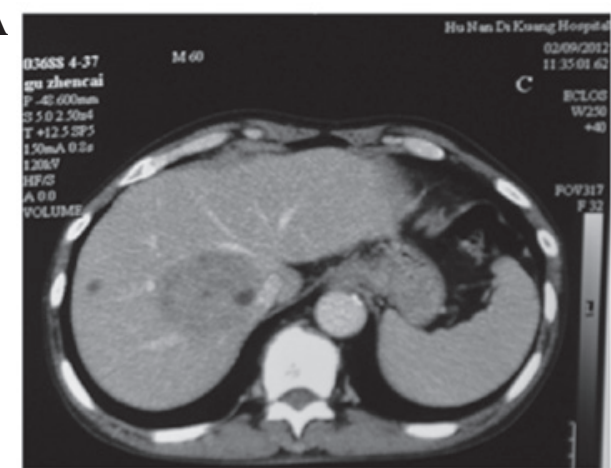

C

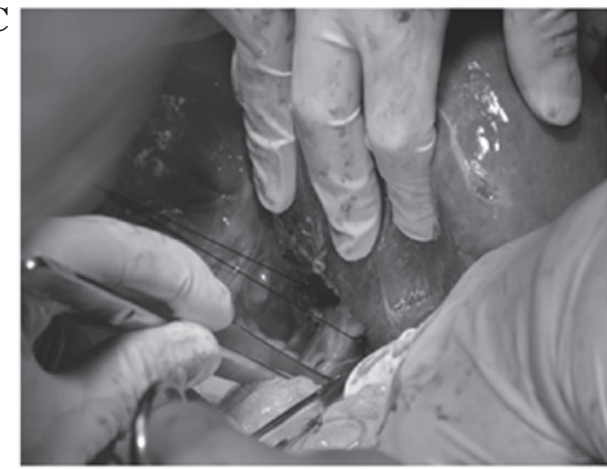

$\mathbf{E}$

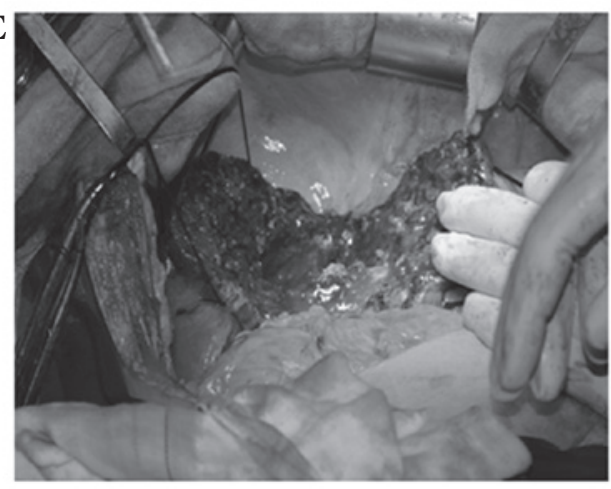

B

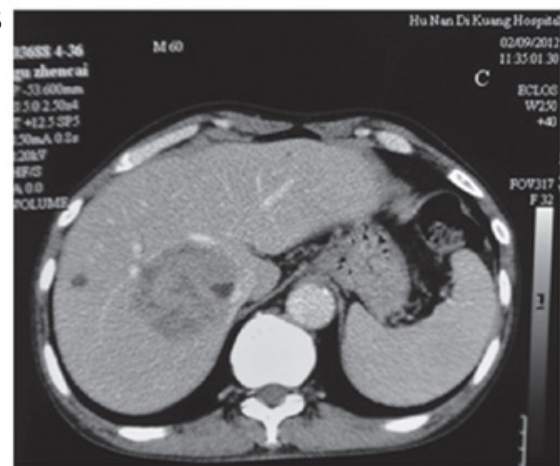

D

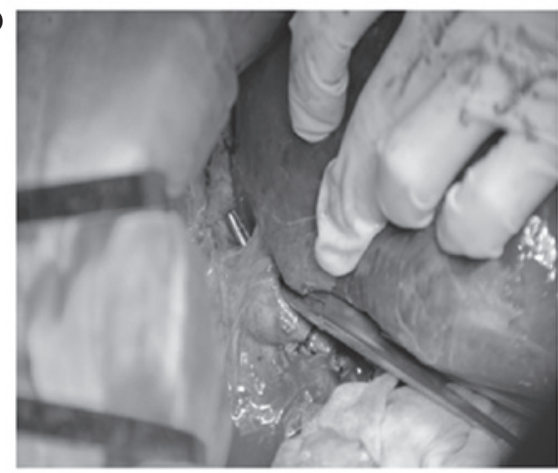

$\mathbf{F}$

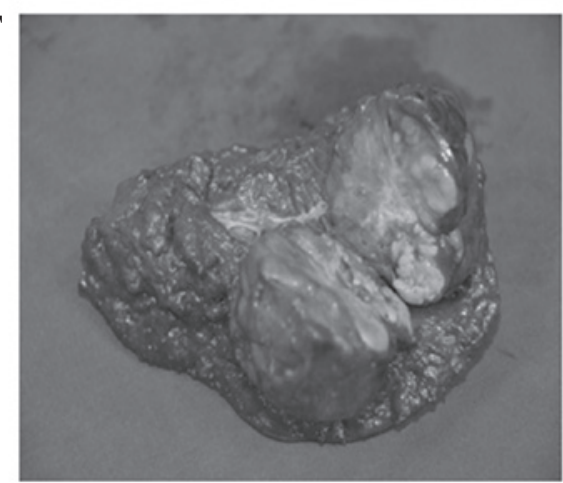

Figure 1. Hepatectomy of segments IV, V, VIII and caudate lobe with cholecystectomy. (A and B) Computed tomography revealed a centrally located hepatocellular carcinoma invading the caudate lobe. (C) A long suture was placed behind the right hepatic vein. (D) The trunk of the left and middle hepatic veins was exposed and the middle hepatic vein was ligated. (E) Postoperative view of the liver. (F) Excised tumor specimen.

pathologically diagnosed primary HCC were included in this study. Ten of the tumors were completely encapsulated, whereas the remaining 14 tumors were not or only partially encapsulated. Of the 24 patients, 19 were hepatitis B surface antigen (HBsAg)-positive, 2 were hepatitis $\mathrm{C}$ virus (HCV)-positive and 2 patients were both $\mathrm{HBsAg-}$ and HCV-positive. All 24 patients were cirrhotic, with the cirrhosis in 18 patients being rated as mild-to-moderate, in 4 patients as severe, while the remaining 2 patients had no obvious liver cirrhosis. Six of these patients also had mild-to-moderate esophageal varices. The $\alpha$-fetoprotein levels, which may be elevated in a subset of HCC patients and are useful for monitoring response to treatment and as an early screening test (13), were $>400 \mu \mathrm{g} / \mathrm{ml}$ in 19 patients. The median tumor diameter was $6.3 \mathrm{~cm}$ (range, $2-14 \mathrm{~cm}$ ). According to the Child-Pugh classification, 21 patients were classed as $\mathrm{A}$ and the remaining 3 patients were classed as B. Child-Pugh class B patients were not subjected to mesohepatectomy until their scores had decreased to A with treatment $(14,15)$. All the patients were administered preop- erative liver-protecting and anticoagulation treatments for 4-5 days prior to mesohepatectomy.

Surgical procedure applied for the isolation of the hepatic artery, portal vein, bile duct and hepatic vein from the tumor. Under general anesthesia, the mesohepatectomy was initiated with a right subcostal or a bilateral subcostal incision with midline extension, or a chevron incision. After the operative field was exposed sufficiently by retractor traction, the texture of the liver, the degree of severity of the cirrhosis and the size and location of the tumors were probed using the fingers. The round, falciform, right triangle, coronary, caudate and hepatocolic ligaments were incised to fully mobilize the right lobe of the liver. After the right side of the hepatic inferior vena cava was separated and exposed, the short hepatic veins were ligated. Subsequently, the hepatic artery, portal vein and bile duct were separated from the tumor. A long suture was placed behind the right hepatic vein to block the hepatoduodenal ligament. The perihepatic ligaments and the bare area of the right lobe of the liver were divided from top to bottom, up to the 

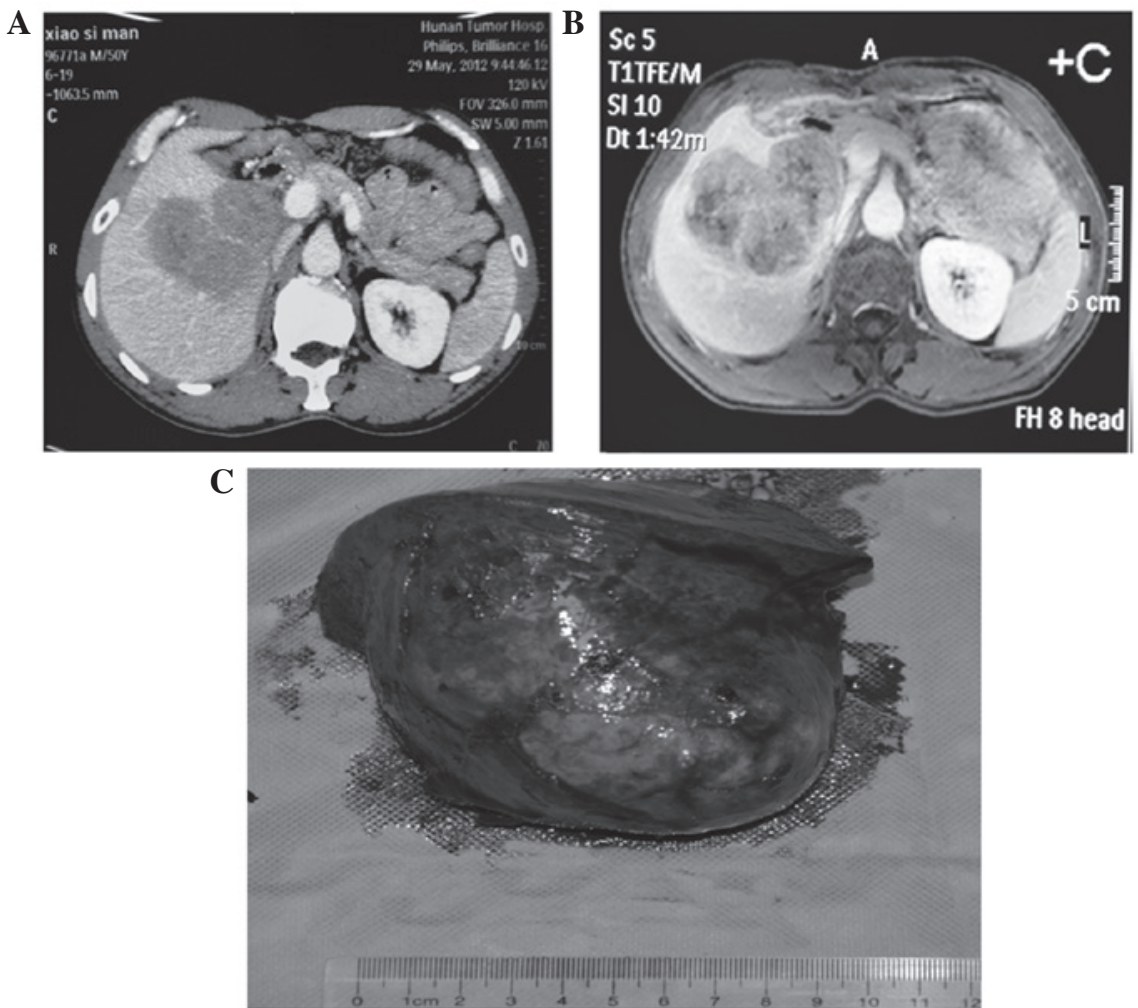

Figure 2. Hepatectomy of segments IV, V and VIII with cholecystectomy. (A and B) Computed tomography revealed a centrally located hepatocellular carcinoma. (C) Excised tumor specimen with complete capsule.
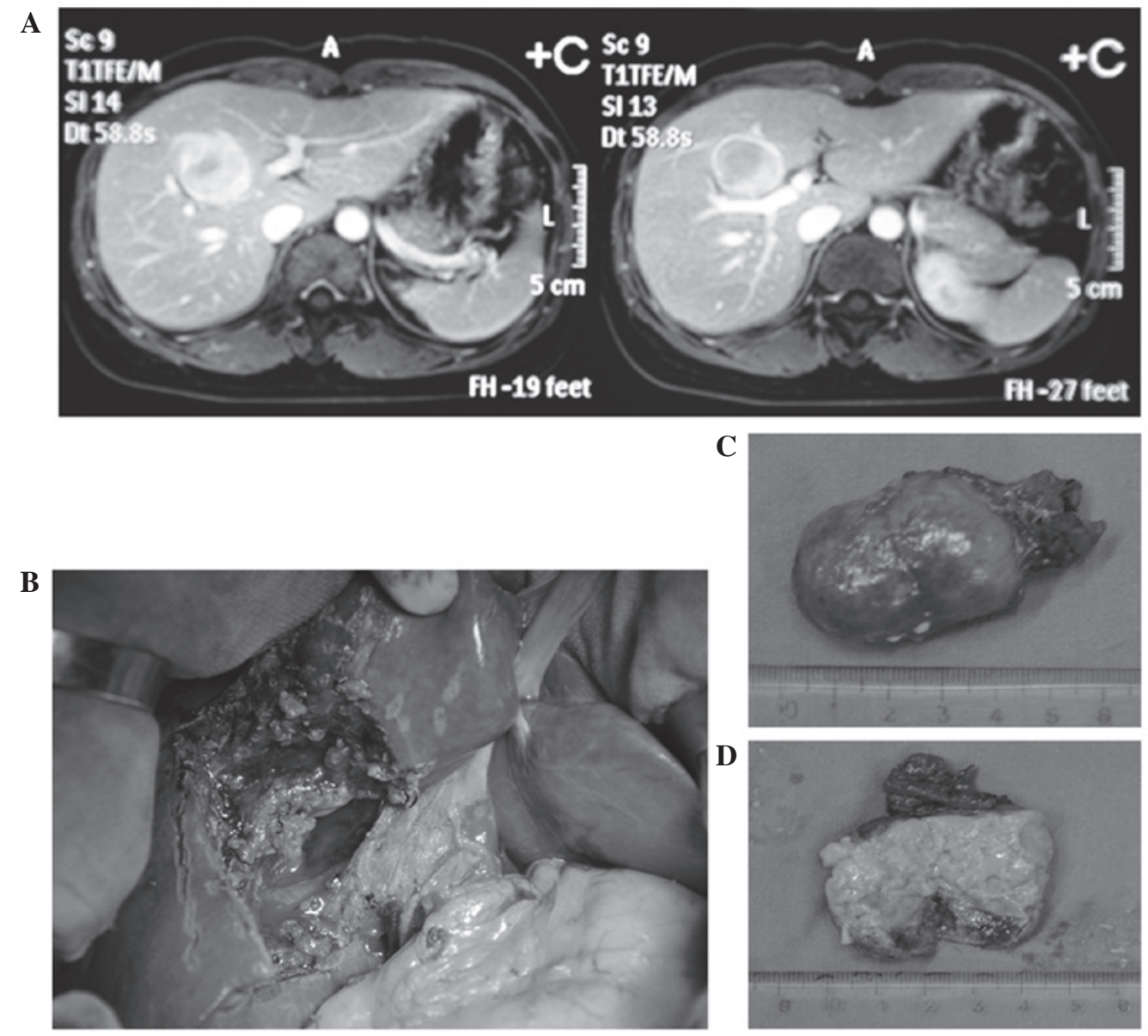

Figure 3. Hepatectomy of segments IVb, V and VIII. (A) Magnetic resonance imaging revealed a centrally located hepatocellular carcinoma compressing the inferior vena cava, hepatic portal blood vessels and bile ducts. (B) Postoperative view of the liver. (C and D) Excised tumor specimen. 
inferior vena cava. The hepatic vena cava gap was separated from top to bottom, up to $3 \sim 4 \mathrm{~cm}$ (Fig. 1), using a right angle clamp. The anterior, left, right and posterior walls of the right hepatic vein were fully exposed to separate and suspend the right hepatic vein with a vascular suture. The left hepatic lobe was mobilized up to the root of the left hepatic vein and the liver cavity was expanded to $3-4 \mathrm{~cm}$. The common trunk of the left and right hepatic veins was divided and vertically clamped with a Simpson clamp (2).

Mesohepatectomy and resectional surface treatment. Intraoperative ultrasonography (US) was used to precisely locate the hepatic veins (15). The hepatic venous circulation was reconstituted according to the preoperative computed tomography (CT) examination. Using an electric or ultrasonic knife, an incision of 2-3 cm in depth was performed in the liver parenchyma along the middle hepatic vein; the vein was separated, ligated and severed between its junction with the inferior vena cava and the common trunk of the left and right hepatic veins. During this process, the right hepatic vein should be well protected. Following complete removal of the middle lobe, bleeding and oozing from the cross sections was fully controlled by clipping or ligating the bleeding sites and biliary leaks, but the resectional surfaces were not sutured compulsively. Of the 24 patients, 8 underwent hepatectomy under intraoperative US guidance.

The surgical approach was selected based on the location and size of the tumor. The blood flow through the hepatic artery and portal vein was intermittently blocked for $20 \mathrm{~min}$, restored for $5 \mathrm{~min}$ and then the cycle was repeated. The blood inflow from the hepatic artery and portal vein in one patient was interrupted 3 times. The average occlusion time was $23 \mathrm{~min}$ (15-60 min). The right hepatic vein and the common trunk of the left and right hepatic veins were clamped with a Satinsky clamp to block the inflow of blood from the hepatic veins, which significantly improved the safety and simplicity of the operative procedure. Occlusion of the hepatic artery, portal vein and bile duct was performed on 17 patients. While 14 of the 17 cases were subjected to occlusion once, 2 patients were subjected to occlusion twice and 1 patient was treated with occlusion 3 times, whereas 4 patients did not undergo occlusion. Total hepatic vascular exclusion (HVE) was performed on 3 patients, once in each patient.

\section{Results}

Procedures. Of the 24 patients, 9 underwent hepatectomy of Couinaud's segments IV, V and VIII with concurrent cholecystectomy (Figs. 1 and 2); 8 underwent hepatectomy of segments IVb, V and VIII (Fig. 3), of whom 7 also received a cholecystectomy (Fig. 4); 4 underwent hepatectomy of segments IVa, V and VIII; and 3 underwent hepatectomy of segments I, IV, V and VIII with concurrent cholecystectomy (Fig. 5).

All the tumors were completely excised and there was no intraoperative mortality. The average intraoperative blood loss was $480 \mathrm{ml}$ (range, 200-2,200 ml). The serum alanine aminotransferase levels returned to normal or preoperative levels in $11 \pm 3$ days post-operation. The total bilirubin decreased to normal or preoperative levels in $8 \pm 2$ days post-operation.
The incidence of complications associated with the operation was $33 \%$ (8/24), including 4 cases of reactive pleural effusion, 3 of biliary fistula and 1 of upper gastrointestinal bleeding. Of the 4 patients with reactive pleural effusion, 2 were treated by pleural puncture and the other 2 were received symptomatic treatment. Patients with other complications also received symptomatic treatment. A total of 23 of the 24 patients $(96 \%)$ were followed up postoperatively. The 1- and 3-year overall survival rates of these patients were 76 and $46 \%$, respectively.

\section{Discussion}

The middle segments of the liver include the medial section of the left lobe and the anterior section of the right lobe. The visceral surface of the middle lobe of the liver is adjacent to the entrance of the hepatic artery, portal vein and bile duct and its diaphragmatic surface is adjacent to the entrance of the hepatic veins, where they merge into the inferior vena cava. The dorsal side of the middle lobe of the liver is where 15 short hepatic veins empty into the inferior vena cava. Due to the unique anatomical location of centrally located HCC, minimally invasive methods such as RFA, cryotherapy and microwave ablation may readily cause heat injuries to the surrounding blood vessels (2-4). Centrally located HCCs tend to invade blood vessels and lead to intrahepatic or extrahepatic metastasis. Therefore, for patients with centrally located $\mathrm{HCC}$, provided their general medical conditions allow surgical intervention and they do not have any concurrent heart, lung, kidney and brain disease or significant severe liver dysfunction, surgical resection is the first choice of treatment, despite a high degree of difficulty and the risks associated with this technically demanding procedure (6). Centrally located HCCs are deeply located, closely adjacent to several major blood vessels and bile ducts; therefore, the selection of the most appropriate surgical approach is crucial for treatment success.

A prerequisite for any successful mesohepatectomy is a well-exposed operation field. To obtain such a field, a bilateral subcostal chevron incision should be adopted, with traction using an all-round retractor. Furthermore, surgeons have to be familiar with the liver anatomy and vascular distribution. During the operation, hepatic vein flow should be accurately assessed by combination of enhanced hepatic CT and magnetic resonance imaging. The hepatic artery, portal vein and bile duct should be protected to avoid any damage and the hepatic arteriovenous vessels of the right and middle lobes of the liver should be fully preserved (16). Injuries in hepatic venous vessels should be repaired with vascular sutures. The wedge area of the middle lobe of the liver is formed by the middle point of the gallbladder bed and the edge of the superior and inferior vena cava. The majority of HCC cases are also complicated by post-hepatitis cirrhosis, fibrosis, compensatory hyperplasia and displacement; therefore, it may be difficult to accurately locate the area to be resected. Intraoperative US is routinely used to probe the middle hepatic vein to determine the boundaries of the middle lobe and the affected area to be removed (15). In our study, the cancer was successfully localized by intraoperative US in 8 patients. Finally, a suitable blockage of blood inflow is key to a successful mesohepatectomy and postoperative patient recovery. In case of massive intraoperative bleeding, it is extremely difficult to protect these major blood vessels, bile ducts and residual liver. 
A
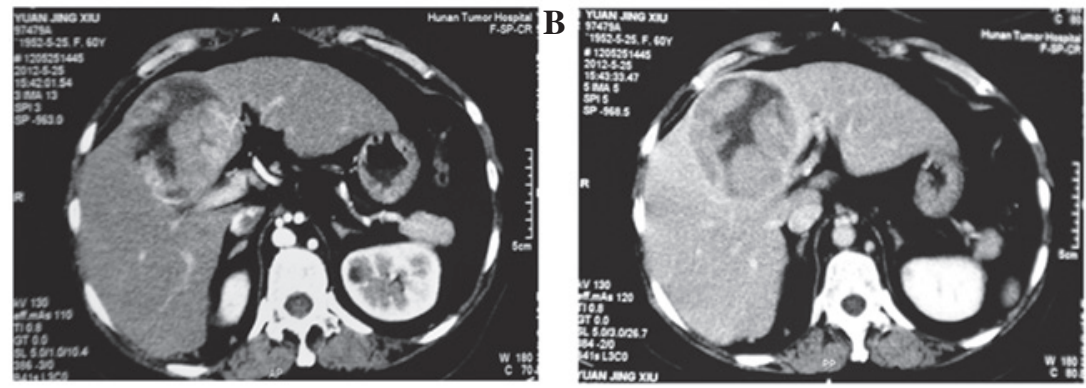

C

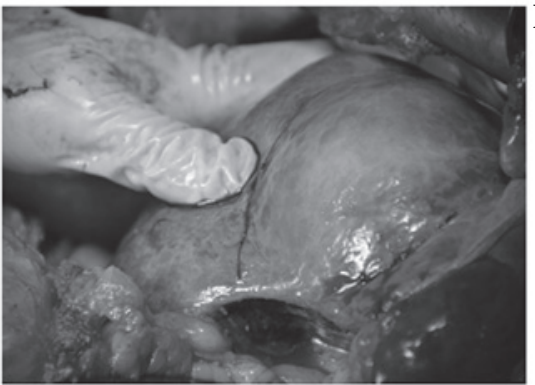

D

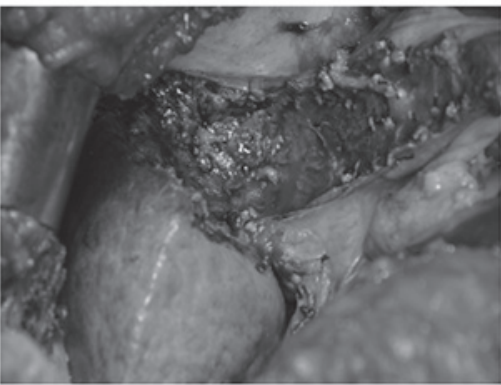

$\mathbf{E}$

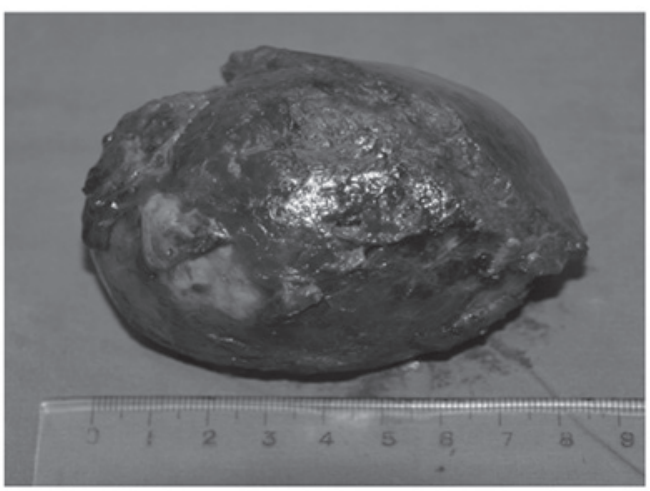

Figure 4. Hepatectomy of segments IVb, V and VIII combined with cholecystectomy. (A and B) Computed tomography revealed a centrally located hepatocellular carcinoma. (C and D) Pre- and postoperative view of the liver. (E) Excised tumor specimen with complete capsule.
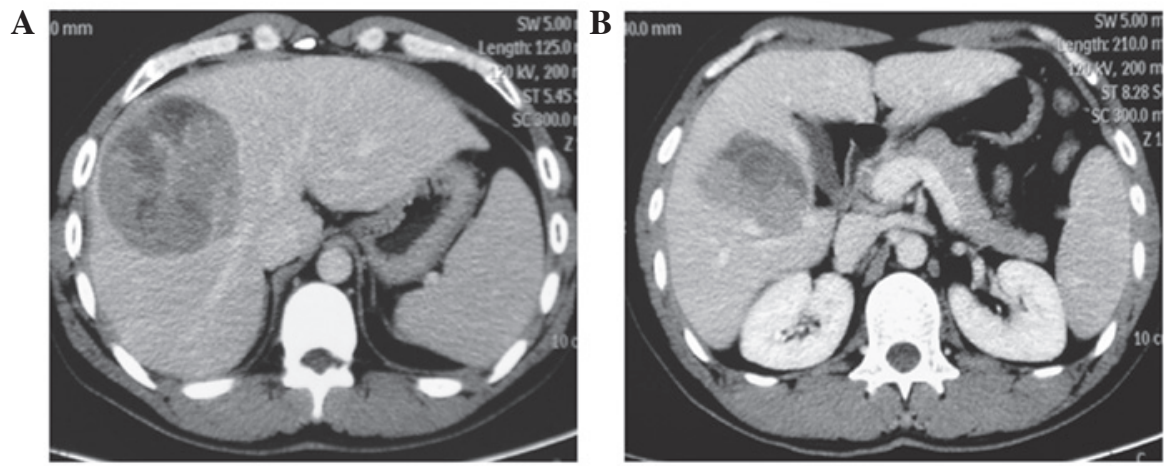

C
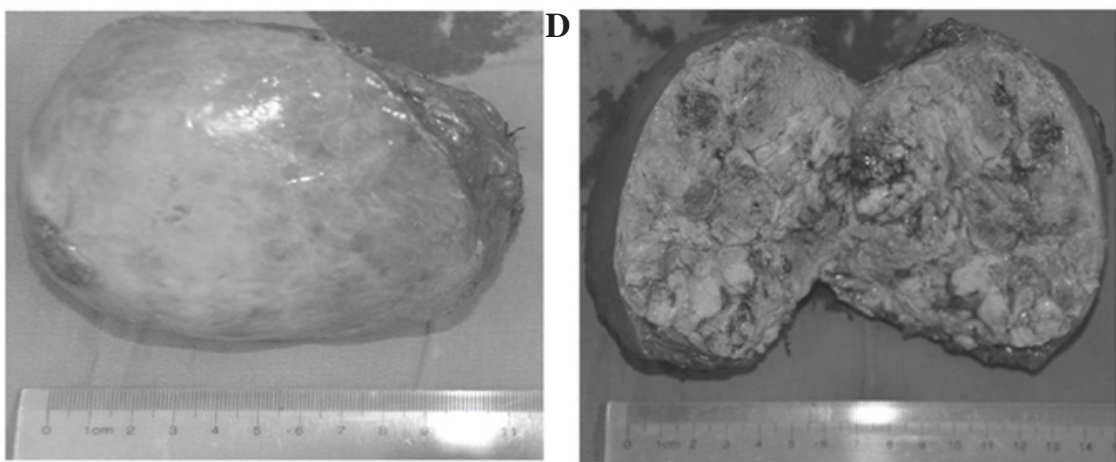

Figure 5. Hepatectomy of segments VI, VII and VIII. (A and B) Computed tomography revealed a centrally located massive hepatocellular carcinoma invading the caudate lobe. (C) Tumor specimen with complete capsule. (D) Excised tumor specimen. 
The middle lobe of the liver is closely adjacent to several major blood vessels. Once uncontrollable massive hemorrhage occurs, injuries to these blood vessels by hasty clamping or blind suturing is unavoidable and these injuries may lead to further severe complications. To reduce intraoperative hemorrhage, we adopted the Pringle maneuver to interrupt the blood flow through the hepatic artery, portal vein and total HVE (8). We also adopted the HVE method reported by Curley et al (2) to selectively separate the hepatic veins and block the inflow of blood. The Pringle maneuver was used during hepatectomy on 18 patients, while HVE was used on 4 patients. The rate of successful inflow occlusion was $83.33 \%$ (20/24). Intermittent inflow occlusion may effectively reduce liver damage caused by ischemia-reperfusion (16). Blockage of blood inflow through the hepatic veins at the right hepatic vein and the common trunk of left and right hepatic veins have significantly improved the safety and simplicity of the operative procedure.

Generally, it is difficult to suture the resectional surfaces of centrally located HCC, since its base consists of the vena cava, portal vein, hepatic artery and bile duct and its bilateral sections are adjacent to the right and left hepatic veins (8). To avoid injuries to the porta hepatis, the suture needle should not be pricked into the liver parenchyma too deeply. We recommend that the resectional surfaces should be left open after the bleeding is controlled. The hepatic resectional surfaces should not be sutured compulsively to avoid compression of the left and right hepatic veins; otherwise, the hepatic venous flow may be interrupted (17). We used biological fibrin glue or styptic powder on the resectional surfaces and the surface was further covered with absorbable hemostatic gauze. For patients with suspected bile duct injuries, the bile ducts were cut open to place a T-tube drain. External T-tube drainage was established in 7 patients (18). One month after the placement, the T-tubes were smoothly pulled out under cholangiographic guidance. Of the 24 patients, 1 was subjected to hepatectomy of segments II, III and VI and Roux-en-Y choledojejunostomy of the right bile duct due to invasion of the left bile duct by recurrent cancer. For resection of centrally located HCC, an incision was performed at the entrance of the hepatic vein and extended further towards the two lateral sides. After the hepatic artery, portal vein and bile duct were clearly exposed, the incision was extended to the entrance of the hepatic artery, portal vein, bile duct, where it was terminated. The openings between the two lateral incisions were kept wide at the top and narrow at the bottom to avoid injuries to the blood vessels and bile ducts. Lang et al (5) also suggested that mesohepatectomy is a safe and effective operative procedure for the treatment of centrally located HCC complicated by hepatitis or cirrhosis.

In summary, despite the unique anatomical location of centrally located HCC and technical complexity of the operating procedure, mesohepatectomy is an effective surgical treatment for patients with centrally located HCCs. The keys for a successful mesohepatectomy include proper preoperative assessment and preparation, precise resection along the middle hepatic vein and suitable inflow occlusion. Mesohepatectomy may be particularly beneficial or even curative for HCC patients with post-hepatitis cirrhosis, as this procedure preserves more functional liver parenchyma compared to conventional or extended lobectomy and reduces the incidence of liver failure. Therefore, we recommend mesohepatectomy as treatment for patients with centrally located HCC with concomitant cirrhosis.

\section{References}

1. Hu RH, Lee PH, Chang YC, Ho MC and Yu SC: Treatment of centrally located hepatocellular carcinoma with central hepatectomy. Surgery 133: 251-256, 2003.

2. Curley SA, Izzo F, Ellis LM, Nicolas Vauthey $J$ and Vallone P: Radiofrequency ablation of hepatocellular cancer in 110 patients with cirrhosis. Ann Surg 232: 381-391, 2000.

3. McGahan JP, Brock JM, Tesluk H, Gu WZ, Schneider P and Browning PD: Hepatic ablation with use of radio-frequency electrocautery in the animal model. J Vasc Interv Radiol 3: 291-297, 1992.

4. Tateishi R, Shiina S, Teratani T, et al: Percutaneous radiofrequency ablation for hepatocellular carcinoma. An analysis of 1000 cases. Cancer 103: 1201-1209, 2005.

5. Lang H, Broelsch CE, Bertona $\mathrm{C}$ and Bourquain $\mathrm{H}$ : Extended left hepatectomy with an inferior right liver vein: improved operation planning by $3-\mathrm{D}$ reconstruction and computer-assisted imaging. J Am Coll Surg 205: 626-627, 2007.

6. Poon RT, Fan ST, Lo CM, et al: Extended hepatic resection for hepatocellular carcinoma in patients with cirrhosis: is it justified? Ann Surg 236: 602-611, 2002.

7. Chedid AD, Chedid MF, Kruel CR, Girardi FM and Krue CD: Extended right hepatectomy with total caudate lobe resection and biliary tree resection for a large colorectal liver metastasis involving both the right and left hepatic lobes and the umbilical fissure: a case report. Am Surg 71: 447-449, 2005.

8. Scudamore CH, Buczkowski AK, Shayan H, Ho SG, Legiehn GM, Chung SW and Owen DA: Mesohepatectomy. Am J Surg 179: 356-360, 2000.

9. Vauthey JN, Pawlik TM, Abdalla EK, et al: Is extended hepatectomy for hepatobiliary malignancy justified? Ann Surg 239: 722-730; discussion 730-732, 2004.

10. Mehrabi A, Mood ZA, Roshanaei N, et al: Mesohepatectomy as an option for the treatment of central liver tumors. J Am Coll Surg 207: 499-509, 2008.

11. Sotiropoulos GC, Lang H, Molmenti EP, Kaiser GM, Paul A and Broelsch CE: Partial or complete mesohepatectomy combined with resection of the hilar bifurcation in cases of Klatskin tumors: a reasonable strategy? Am J Surg 198: 297-298, 2009.

12. Wu CC, Ho WL, Chen JT, et al: Mesohepatectomy for centrally located hepatocellular carcinoma: an appraisal of a rare procedure. J Am Coll Surg 188: 508-515, 1999.

13. Riaz A, Ryu RK, Kulik LM, et al: Alpha-fetoprotein response after locoregional therapy for hepatocellular carcinoma: oncologic marker of radiologic response, progression and survival. J Clin Oncol 27: 5734-5742, 2009.

14. Ishii $\mathrm{H}$, Ogino $\mathrm{S}$, Ikemoto $\mathrm{K}$, et al: Mesohepatectomy with total caudate lobectomy of the liver for hepatocellular carcinoma. World J Surg Oncol 11: 82, 2013.

15. Kishi Y, Hasegawa K, Sugawara $\mathrm{Y}$ and Kokudo N: Hepatocellular carcinoma: current management and future development-improved outcomes with surgical resection. Int $\mathbf{J}$ Hepatol 2011: 728103, 2011.

16. Yang Y, Fu SY,Lau WY, et al: Selective main portal vein clamping to minimize the risk of recurrence after curative liver resection for hepatocellular carcinoma. Hepatogastroenterology 59: 1560-1565, 2012.

17. Chang YC, Nagasue N, Chen CS and Lin XZ: Simplified hepatic resections with the use of a Chang's needle. Ann Surg 243: 169-172, 2006

18. Soulez G, Lerouge S, Allard L, et al: Vulnerable carotid atherosclerotic plaque creation in a swine model: evaluation of stenosis creation using absorbable and permanent suture in a diabetic dyslipidemic model. J Vasc Interv Radiol 23: 1700-1708, 2012. 